\title{
THE TREE OF SHAPES OF AN IMAGE*
}

\author{
Coloma Ballester ${ }^{1}$, Vicent Caselles ${ }^{1}$ And P. Monasse $^{2}$
}

\begin{abstract}
In [30], Kronrod proves that the connected components of isolevel sets of a continuous function can be endowed with a tree structure. Obviously, the connected components of upper level sets are an inclusion tree, and the same is true for connected components of lower level sets. We prove that in the case of semicontinuous functions, those trees can be merged into a single one, which, following its use in image processing, we call "tree of shapes". This permits us to solve a classical representation problem in mathematical morphology: to represent an image in such a way that maxima and minima can be computationally dealt with simultaneously. We prove the finiteness of the tree when the image is the result of applying any extrema killer (a classical denoising filter in image processing). The shape tree also yields an easy mathematical definition of adaptive image quantization.
\end{abstract}

Mathematics Subject Classification. 68U10, O5C05.

Received February 1, 2002.

\section{INTRODUCTION AND MOTIVATION}

An image, to be processed, must be represented by an adequate model. For example, its Fourier transform is often used for denoising or registration, see [56]. Its decomposition on a wavelet or wavelet packet basis can be used for multiple tasks, the most convincing one being compression, see [31]. These models, totally (in the Fourier case) or partially (in the wavelet case) based on the frequencies of the image, are however dependent on the original image contrast. The same thing can be said of image representations based on edges [25]. While this is not a problem in image compression, it becomes more problematic in image analysis, since we would like any automatic tool to be insensitive to contrast changes, in as much as our vision is [55].

In the same way, most algorithms trying to segment the image into significant regions, i.e., trying to find a partition of the image into essentially homogeneous regions, are generally not contrast invariant, see, for instance $[28,37]$.

Mathematical morphology is able to deal with contrast invariant objects, the most basic ones being the (upper) level sets $[u \geq \lambda]=\{x, u(x) \geq \lambda\}$, or the level lines $\partial[u \geq \lambda]$ of the image $u[32,40-42,45,46]$. They are said to be contrast invariant, since, for any increasing continuous function $g$, the level sets of $g \circ u$ are globally the same as the level sets of $u$, modulo a change of level. Many tasks can be efficiently addressed

Keywords and phrases: Image representation, mathematical morphology, tree structure, level sets.

* We acknowledge partial support by the TMR European project "Viscosity solutions and their applications", reference FMRXCT98-0234 and the CNRS through a PICS project. The first two authors also acknowledge partial support by the PNPGC project, reference BFM2000-0962-C02-01.

1 Univ. Pompeu-Fabra, Passeig de Circumvalació 8, 08003 Barcelona, Spain;

e-mail: coloma.ballester@tecn.upf.es, vicent.caselles@tecn.upf .es

2 CMLA, ENS Cachan, 61 avenue du Président Wilson, 94235 Cachan Cedex, France; e-mail: monasse@cmla.ens-cachan.fr 
by means of morphological methods: contrast enhancement [7,8], filtering [1,22, 48, 49], compression [18, 43], segmentation [27,40,53], intersection [4], or registration [34], to give some examples. Mathematical morphology uses the structure of level sets in a fundamental way: indeed, the upper level sets $[u \geq \lambda]$ are nonincreasing with respect to $\lambda$, and any contrast invariant process can be interpreted as a geometric process acting on level sets, preserving their order. The resulting image is defined so that its level sets are the processed level sets of the original image (each point gets the level of the smallest processed level set containing it).

As objects of interest can be bright, as well as dark, a frequent requirement of mathematical morphology is to have self-dual algorithms, i.e., algorithms which act in the same manner on upper and lower level sets. And here comes a difficulty, self-dual algorithms modify the families of upper and lower level sets. However, each one of these families yields a complete representation of image, and when we modify one, it is not easy to ensure consistency with the other one.

Moreover, as most objects consist of a single piece, we prefer to take as basic objects of the image the connected components of its level sets, and, ask the basic operations to act on this structure. Those operations are called connected filters since they preserve connectedness [33,42,47]. Connected components of upper (or lower) level sets have a natural tree structure given by the inclusion order. Putting both together creates a selfdual, but redundant, representation of the image. Does it exist a self-dual, and non redundant, representation of the image? As this is not possible with both families of connected components of level sets, we shall modify them. The modification used in this paper consists in filling-in its holes. We shall see that, by doing this, we are able to create a single tree of basic objects. This tree structure was first used as an algorithm, but not proved, in [50], and the importance of the role played by holes was not explained.

A very close structure based on level lines, and valid for continuous images, was discovered by Kronrod [30]. However, images have discontinuities, prominently at the edges of objects. The present work can be understood as an extension of Kronrod's tree to semicontinuous images. The tree structure can also be compared to the Morse tree $[19,29]$, but the regularity required for Morse functions is too stringent for images. An adaptation to images was recently proposed under the name of Digital Morse Theory [10], but this representation does not show explicitly the upper or lower level sets. The assumption that images are $B V$ functions, i.e., functions of bounded variation $[2,11,17]$, was introduced to deal with the restoration problem $[9,16,39,54]$. In this model, to define basic objects as connected components of level sets and the corresponding extrema killers, an adapted notion of connectedness, called $M$-connectedness, is proposed in $[3,5]$. Apart from the fact that there are clues hinting that images are not of bounded variation due to texture oscillations [20], the $M$-connectedness will be shown not to yield a tree structure. Thus, we shall restrict ourselves to the case of images as uppersemicontinuous functions, a model which permits discontinuities and the definition of a self-dual tree structure for the shapes of the image. Practical applications of the representation we study here, as well as a fast algorithm to extract it, have been presented in $[35,36]$. Let us finally mention that general uses of a tree structure to design filters are explained in [44].

Let us explain the plan of the paper. Next section is devoted to define the notions of saturation and of holes, and to study its basic properties. In Section 3 we prove that the shapes of an image, defined as the saturation of the connected components of its level sets, have a tree structure and we study it. In Section 4 we prove that the tree is equivalent to the image: the knowledge of the tree is sufficient to reconstruct the image. Section 5 shows the finiteness of the tree structure under hypotheses that are met for digital images. Last section is devoted to an application, indeed, we show how to obtain schematic versions of the image by manipulating its associated tree.

\section{SOME TOPOLOGICAL PRELIMINARIES}

Let $\bar{\Omega}$ be a set homeomorphic to the closed unit ball of $\mathbb{R}^{N}(N \geq 2),\left\{x \in \mathbb{R}^{N},\|x\| \leq 1\right\}$, and $\Omega$ be the interior of $\bar{\Omega}$. Note that, in particular, $\bar{\Omega}$ is compact, connected and locally connected. Moreover, $\bar{\Omega}$ is unicoherent.

Definition 2.1 ([23], Sect. 41, X). A topological space $Z$ is said to be unicoherent if it is connected and for any two closed connected sets $A, B$ in $Z$ such that $Z=A \cup B, A \cap B$ is connected. 
Usually, $\bar{\Omega}$ is a closed rectangle of $\mathbb{R}^{2}$. An image $u$ is a map from $\bar{\Omega}$ to $\mathbb{R}$, supposed to be upper semicontinuous. The lower and upper level sets of $u$ are the sets

$$
[u<\lambda]=\{x \in \bar{\Omega}, u(x)<\lambda\} \quad[u \geq \lambda]=\{x \in \bar{\Omega}, u(x) \geq \lambda\} .
$$

Lower level sets are open, while upper level sets are closed. The asymmetry comes from the assumption that $u$ is upper semicontinuous, and would be inverted if we assumed $u$ to be lower semicontinuous.

Definition 2.2. Let $A \subseteq \bar{\Omega}$. We call holes of $A$ in $\bar{\Omega}$ the components of $\bar{\Omega} \backslash A$. Let $p_{\infty} \in \bar{\Omega} \backslash A$ be a reference point, and let $T$ be the hole of $A$ in $\bar{\Omega}$ containing $p_{\infty}$. We define the saturation of $A$ with respect to $p_{\infty}$ as the set $\bar{\Omega} \backslash T$ and we denote it by $\operatorname{Sat}\left(A, p_{\infty}\right)$. We shall refer to $T$ as the external hole of $A$ and to the other holes of $A$ as its internal holes. By extension, if $p_{\infty} \in A$, by convention we $\operatorname{define} \operatorname{Sat}\left(A, p_{\infty}\right)=\bar{\Omega}$. Note that $\operatorname{Sat}\left(A, p_{\infty}\right)$ is the union of $A$ and its internal holes.

The reference point $p_{\infty}$ acts as a point at infinity. In all what follows, we assume that the point $p_{\infty} \in \bar{\Omega}$ on which the saturations are based is fixed, i.e., all saturations will be computed with respect to $p_{\infty}$. To simplify our notation, we shall write $\operatorname{Sat}(A)$ instead of $\operatorname{Sat}\left(A, p_{\infty}\right)$. We shall also speak of holes of $A$ instead of holes of $A$ in $\bar{\Omega}$.

First, we prove some basic properties of the saturation.

Lemma 2.3. Let $A \subseteq \bar{\Omega}$. If $A$ is open (resp. closed) in $\bar{\Omega}$, then its saturated sets are open (resp. closed) in $\bar{\Omega}$.

Proof. If $A$ is open in $\bar{\Omega}, \bar{\Omega} \backslash \operatorname{Sat}(A)$, being a connected component of a closed set in $\bar{\Omega}$, is also closed in $\bar{\Omega}$. Thus $\operatorname{Sat}(A)$ is open in $\bar{\Omega}$. If $A$ is closed in $\bar{\Omega}, \bar{\Omega} \backslash \operatorname{Sat}(A)$, being a connected component of an open set in $\bar{\Omega}$, is also open, as $\bar{\Omega}$ is locally connected. Thus $\operatorname{Sat}(A)$ is closed in $\bar{\Omega}$.

Lemma 2.4. The saturation Sat(.), as an operator acting on subsets of $\bar{\Omega}$, is

(i) monotone: $A \subseteq B \Rightarrow \operatorname{Sat}(A) \subset \operatorname{Sat}(B)$;

(ii) idempotent: $\operatorname{Sat}[\operatorname{Sat}(A)]=\operatorname{Sat}(A)$.

Proof. The proofs are immediate and we shall skip them.

Lemma 2.5. Let $A \subseteq \bar{\Omega}$ be a connected set and $T$ be a hole of $A$. If $T$ is an internal hole, Sat $T=T$; if $T$ is the external hole, $\operatorname{Sat}(T)=\bar{\Omega}$.

Proof. If $T$ is an internal hole, then $p_{\infty} \notin T$, and, therefore, $\operatorname{Sat}(T) \neq \bar{\Omega}$. Since $T$ is a connected component of $\bar{\Omega} \backslash A$, the set $\bar{\Omega} \backslash T$ is connected ([38], IV.3, Th. 3.3). Hence $\bar{\Omega} \backslash T$ is a hole of $T$ containing $p_{\infty}$, and we conclude that $\operatorname{Sat}(T)=T$. If $T$ is the external hole, then $p_{\infty} \in T$, and we have that $\operatorname{Sat}(T)=\bar{\Omega}$.

We now prove three lemmas that will be useful in the sequel:

Lemma 2.6. If $A \subseteq \bar{\Omega}$ is connected, then $\operatorname{Sat}(A)$ is also connected.

Proof. If $\operatorname{Sat}(A)=\bar{\Omega}$, there is nothing to prove. Otherwise, $\bar{\Omega} \backslash$ Sat $A$ is a connected component of the complement of a connected set $A$, in a connected space $\bar{\Omega}$. Thanks to [23] (Sect. 41,III,5), its complement, i.e., Sat $(A)$, is connected.

The notation used will be the usual one in topology. If $A$ is a set in a topological space, $\stackrel{\circ}{A}, \bar{A}$ and $\partial A$ will denote, respectively, the interior, the closure and the boundary of $A$. If $p \in A$, we denote the connected component of $A$ containing $p$ by $\operatorname{cc}(A, p)$. By $\operatorname{cc}(A)$ we shall denote a connected component of $A$.

Lemma 2.7. Let $A \subseteq \bar{\Omega}$. If $T$ is a hole of $A$, then

$$
\partial T \subseteq \partial A .
$$

As a consequence,

$$
\partial \operatorname{Sat}(A) \subseteq \partial A .
$$


Proof. $T$ is a connected component of $\bar{\Omega} \backslash A$, so $\partial T \subseteq \partial(\bar{\Omega} \backslash A)$ (see [23], Sect. 44, III, 3), proving that $\partial T \subseteq \partial A$. Let $T$ be the external hole of $A$. Then

$$
\partial \operatorname{Sat}(A)=\partial(\bar{\Omega} \backslash \operatorname{Sat}(A))=\partial T \subseteq \partial A .
$$

Lemma 2.8. Let $A \subseteq \bar{\Omega}$ be such that $\operatorname{Sat}(A) \neq \bar{\Omega}$. Then $\operatorname{Sat}(A) \subseteq \operatorname{Sat}(\partial A)$, and, if $A$ is closed, we get $\operatorname{Sat}(A)=\operatorname{Sat}(\partial A)$.

Proof. Suppose first that $A$ is closed. Since $\partial A \subseteq A$, taking the saturation of each member, we have that $\operatorname{Sat}(\partial A) \subseteq \operatorname{Sat}(A)$. To prove the other inclusion, let $C$ be a connected component of $\stackrel{\circ}{A}$. It is easy to see that $C$, as any open connected set, is a hole of $\partial C$. Since $\partial C \subseteq \partial \stackrel{\circ}{A}$ and $C \cap \partial \stackrel{\circ}{A}=\emptyset$, we have that $C$ is open and closed in $\bar{\Omega} \backslash \partial \stackrel{\circ}{A}$. Since $C$ is also connected, then $C$ is a hole of $\partial \stackrel{\circ}{A}$, and, therefore, $C \subseteq \operatorname{Sat}(\partial \stackrel{\circ}{A})$. Now, using that

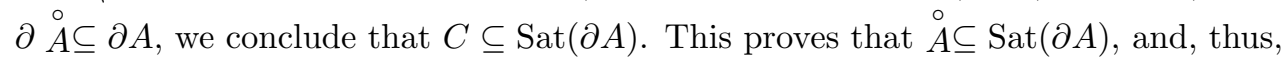

$$
A=\stackrel{\circ}{A} \cup \partial A \subseteq \operatorname{Sat}(\partial A) .
$$

Finally, taking saturations, we obtain $\operatorname{Sat}(A) \subseteq \operatorname{Sat}(\partial A)$.

For an arbitrary set $A, A \subseteq \bar{A}$ implies that $\operatorname{Sat}(A) \subseteq \operatorname{Sat}(\bar{A})$. If $\operatorname{Sat}(\bar{A}) \neq \bar{\Omega}$, the first part of the proof applies to $\bar{A}$ and we obtain that $\operatorname{Sat}(A) \subseteq \operatorname{Sat}(\bar{A})=\operatorname{Sat}(\partial \bar{A})$. Finally, from $\partial \bar{A} \subseteq \partial A$, we conclude that $\operatorname{Sat}(A) \subseteq \operatorname{Sat}(\partial A)$.

Now, the remaining case is $\operatorname{Sat}(\bar{A})=\bar{\Omega}$. If, in addition, we have that $\operatorname{Sat}(\partial A)=\bar{\Omega}$, the result is obvious. Otherwise, let $E=\bar{\Omega} \backslash \operatorname{Sat}(\partial A) \neq \emptyset$. Then $E$ is a hole of $\partial A$, hence, it cannot meet both $A$ and $\bar{\Omega} \backslash A$. If $E \subseteq A$, then Sat $E \subseteq \operatorname{Sat} A \neq \bar{\Omega}$, so that $\operatorname{Sat}(E)=\emptyset$, a contradiction, since $E \subseteq \operatorname{Sat}(E)$. Thus, $E$ is contained in a hole $T$ of $A, T$ being not an internal hole, since, otherwise, $\operatorname{Sat}(E) \subseteq \operatorname{Sat}(T) \subseteq \operatorname{Sat}(A)$, which is again impossible. Therefore, $T$ is the external hole of $A$, and $E \subseteq T$ amounts to

$$
\bar{\Omega} \backslash \operatorname{Sat}(\partial A) \subseteq \bar{\Omega} \backslash \operatorname{Sat}(A),
$$

which proves the expected result.

\section{THE TREE OF SHAPES}

Definition 3.1. Given an image $u$, we call shapes of inferior (resp. superior) type the sets

$$
\operatorname{Sat}(\operatorname{cc}([u<\mu], x)) \quad(\operatorname{resp} . \operatorname{Sat}(\operatorname{cc}([u \geq \lambda], x))),
$$

where $\lambda, \mu \in \mathbb{R}, x \in \bar{\Omega}$. We call shape of $u$ any shape of inferior or superior type. We denote by $\mathcal{S}(u)$ the shapes of $u$.

Having fixed the point $p_{\infty}$ on which the saturation is based, the shapes of the image depend on $p_{\infty}$. If we choose $p_{\infty} \in \partial \Omega$ and we assume that the function $u$ is constant in a neighborhood of $\partial \Omega$, then we may consider that our notion of shape becomes intrinsic.

We note that, by definition, shapes of superior type are closed, while shapes of inferior type are open. Since shapes are connected, the only shapes of both types are $\emptyset$ and $\bar{\Omega}$.

The rest of the section is devoted to prove that shapes have a kind of tree structure, and to define common features of this tree, like branches and leaves. 


\subsection{Tree structure of the set of shapes}

Lemma 3.2. Let $A, B \subseteq \bar{\Omega}$ be connected sets such that $A \cap B=\emptyset$. Then $\operatorname{Sat}(A)$ and $\operatorname{Sat}(B)$ are either disjoint or nested.

Proof. The result is obvious if one of the saturations coincides with $\bar{\Omega}$. Thus, we may suppose that $A$ and $B$ have each an external hole. Then, being connected, $A$ is included in a hole $T$ of $B$. If $T$ is an internal hole, we get

$$
\operatorname{Sat}(A) \subseteq \operatorname{Sat}(T)=T \subseteq \operatorname{Sat} B
$$

If $T$ is the external hole of $B$, then $B$ is also included in a hole $H$ of $A$. If $H$ is an internal hole of $A$, the same proof above applies and we get $\operatorname{Sat}(B) \subseteq \operatorname{Sat}(A)$. If $H$ is the external hole of $A$, no internal hole of $A$ meets $B$, and, thus, $\operatorname{Sat}(A)$ is in the exterior hole of $B$. Therefore, no internal hole of $B$ meets $\operatorname{Sat}(A)$, implying that $\operatorname{Sat}(A) \cap \operatorname{Sat}(B)=\emptyset$.

Theorem 3.3. Any two shapes are either disjoint or nested.

Proof. Let $A=\operatorname{Sat}(\tilde{A})$ and $B=\operatorname{Sat}(\tilde{B}), \tilde{A}$ and $\tilde{B}$ being connected components of level sets of $u$.

If $\tilde{A}$ and $\tilde{B}$ are of the same type, then they are either nested or disjoint. In the first case, $A$ and $B$ are nested because Sat is a monotone operator, while, in the second case, Lemma 3.2 permits to conclude. Thus, we may assume that $\tilde{A}, \tilde{B}$ are of different type and $\tilde{A} \cap \tilde{B} \neq \emptyset$. Let $x \in \tilde{A} \cap \tilde{B}$. By interchanging names, if necessary, we may assume that $\tilde{A}=\operatorname{cc}([u \geq \lambda])$ and $\tilde{B}=\operatorname{cc}([u<\mu])$. Observe that $\lambda \leq u(x)<\mu$, hence $\lambda<\mu$.

The set $\tilde{B}$, being a connected component of $[u<\mu]$, is open in $\bar{\Omega}$ and closed in $[u<\mu]$, hence,

$$
[u<\mu] \cap \partial \tilde{B}=[u<\mu] \cap(\tilde{B} \backslash \tilde{B})=\emptyset .
$$

This proves that $\partial \tilde{B} \subseteq[u \geq \mu] \subseteq[u \geq \lambda]$ and, according to Lemma 2.7, $\partial B \subseteq \partial \tilde{B} \subseteq[u \geq \lambda]$.

Since $A$ is connected, if $\partial B \cap A=\emptyset$, we have that either $A \subseteq B$ or $A \subseteq \bar{\Omega} \backslash \bar{B}$, the last case being impossible because $A \cap B \neq \emptyset$. Thus, we may assume that $\partial B \cap A \neq \emptyset$. First, we observe that $\partial B$ is connected. Indeed, if $T$ is the external hole of $B$, the connectedness of $\partial B$ follows from the identity

$$
\partial B=\bar{B} \cap \bar{T}
$$

and the unicoherency of $\bar{\Omega}$, since $\bar{B}$ and $\bar{T}$ are connected, being the closure of connected sets.

From the above, we conclude that $\partial B$ is contained in a connected component $C$ of $[u \geq \lambda]$. If $C \cap \tilde{A}=\emptyset$, then $C$ is in a hole of $\tilde{A}$, but not in the external one as $\partial B \cap A \neq \emptyset$. The other case being $C=\tilde{A}$, we conclude that, in any case, we have that $\partial B \subseteq A$. Now, using Lemma 2.8, we obtain

$$
B=\operatorname{Sat}(B) \subseteq \operatorname{Sat}(\partial B) \subseteq \operatorname{Sat}(A)=A
$$

concluding the proof.

Theorem 3.3 is the key to prove that the inclusion of subsets induces a tree structure on the set of shapes. The root of the tree is

$$
\bar{\Omega}=\operatorname{Sat}\left(\left[u<1+\max _{\bar{\Omega}} u\right]\right),
$$

and there is no loop: if $A, B_{1}, B_{2}, C$ are shapes and $A \subseteq B_{i} \subseteq C, i=1,2$, then $B_{1} \cap B_{2} \neq \emptyset$, hence the sets $B_{1}$ and $B_{2}$ must be nested. 


\subsection{Branches, monotone sections and leaves}

Definition 3.4. Let $A \subseteq B \subseteq \bar{\Omega}$. We define $[A, B]$ as the interval of shapes between $A$ and $B$, i.e.,

$$
[A, B]=\{S: S \in \mathcal{S}(u), A \subseteq S \subseteq B\}
$$

We also define

$$
\inf [A, B]=\bigcap_{S \in[A, B]} S \text { and } \sup [A, B]=\bigcup_{S \in[A, B]} S .
$$

We say that $T$ is a limit shape if it is the infimum or the supremum of a nonempty interval of shapes.

Observe that, if $A \subseteq B \subseteq \bar{\Omega}$, then $[A, B]=[\inf [A, B], \sup [A, B]]$. Thus, when considering an interval, we may always assume that its extreme sets are limit shapes.

Definition 3.5. Let $B \subseteq \bar{\Omega}$. We say that $B$ contains a bifurcation if there exist $S, T \in \mathcal{S}(u)$ such that $S, T \subseteq B$ and $S \cap T=\emptyset$. Let $A \subseteq B \subseteq \bar{\Omega}$. We say that there is a bifurcation between $A$ and $B$ if $\inf [A, B] \neq \emptyset$ and there is $S \in \mathcal{S}(u)$ such that $S \subseteq B$ and $S \cap \inf [A, B]=\emptyset$.

Definition 3.6. Let $A \subseteq B \subseteq \bar{\Omega}$. We say that $[A, B]$ is a branch of the tree of shapes of $u$ if there is no bifurcation between $A$ and $B$. We say that $[A, B]$ is a monotone section if $[A, B]$ is a branch with all shapes of the same type.

We say that a branch $[A, B]$ (resp., a monotone section) contains $x \in \bar{\Omega}$ if there is a shape $S \in[A, B]$ such that $x \in S$.

Definition 3.7. Let $A \subseteq B \subseteq \bar{\Omega}$. We say that $[A, B]$ has a definite type if all its shapes are of the same type.

Definition 3.8. Let $x \in \bar{\Omega}$. We define $S_{x}$ as the smallest limit shape containing $x$, i.e.,

$$
S_{x}=\inf [\{x\}, \bar{\Omega}]=\bigcap_{S \in \mathcal{S}(u), x \in S} S .
$$

The upper branch at $x$ is the set

$$
\mathcal{B}_{x}=\left\{S: S \in \mathcal{S}(u), x \in S, \text { and }\left[S_{x}, S\right] \text { is a branch }\right\}
$$

Definition 3.9. We call leaf of the tree of shapes of $u$, or simply, a leaf, any limit shape $L=\inf [A, B]$ containing no shape.

These various notions are illustrated in Figure 1.

Proposition 3.10. Any two limit shapes are either nested or disjoint.

Proof. Assume first that $T$ is a shape and $S$ is a limit shape such that $T \cap S \neq \emptyset$. Suppose that $S=\inf [A, B]$. Then $T \cap R \neq \emptyset$ for all $R \in[A, B]$. By Theorem 3.3, for any $R \in[A, B]$, either $T \subseteq R$ or $R \subseteq T$. Then, either there is $R \in[A, B]$ such that $R \subseteq T$, or $T \subseteq R$, for all $R \in[A, B]$. In the first case, we have that $S=\inf [A, B] \subseteq T$. In the second one, $T \subseteq \inf [A, B]=S$.

Suppose that $S=\sup [A, B]$. Since $T \cap S \neq \emptyset$, there is $R \in[A, B]$ such that $T \cap R \neq \emptyset$. Observe that $S=\sup [R, B]$ and $T \cap Q \neq \emptyset$ for all $Q \in[R, B]$. By Theorem 3.3, for any $Q \in[R, B]$, either $T \subseteq Q$ or $Q \subseteq T$. Then, either there is $Q \in[R, B]$ such that $T \subseteq Q$, or $Q \subseteq T$, for all $Q \in[R, B]$. In the first case, we have that $T \subseteq \sup [R, B]=S$. In the second one, $S=\sup [A, B] \subseteq T$.

Now, we suppose that both $S$ and $T$ are limit shapes. First, assume that $S=\inf [A, B]$. If $S \cap T \neq \emptyset$, then $R \cap T \neq \emptyset$ for all $R \in[A, B]$. Then, either there is some $R \in[A, B]$ such that $R \subseteq T$, or $T \subseteq R$ for all $R \in[A, B]$. In the first case, $S \subseteq T$, in the second, $T \subseteq S$. Now, assume that $S=\sup [A, B]$. If $S \cap T \neq \emptyset$, then there is some $R \in[A, B]$ such that $R \cap T \neq \emptyset$. Observe that $S=\sup [R, B]$ and $T \cap Q \neq \emptyset$ for all $Q \in[R, B]$. 


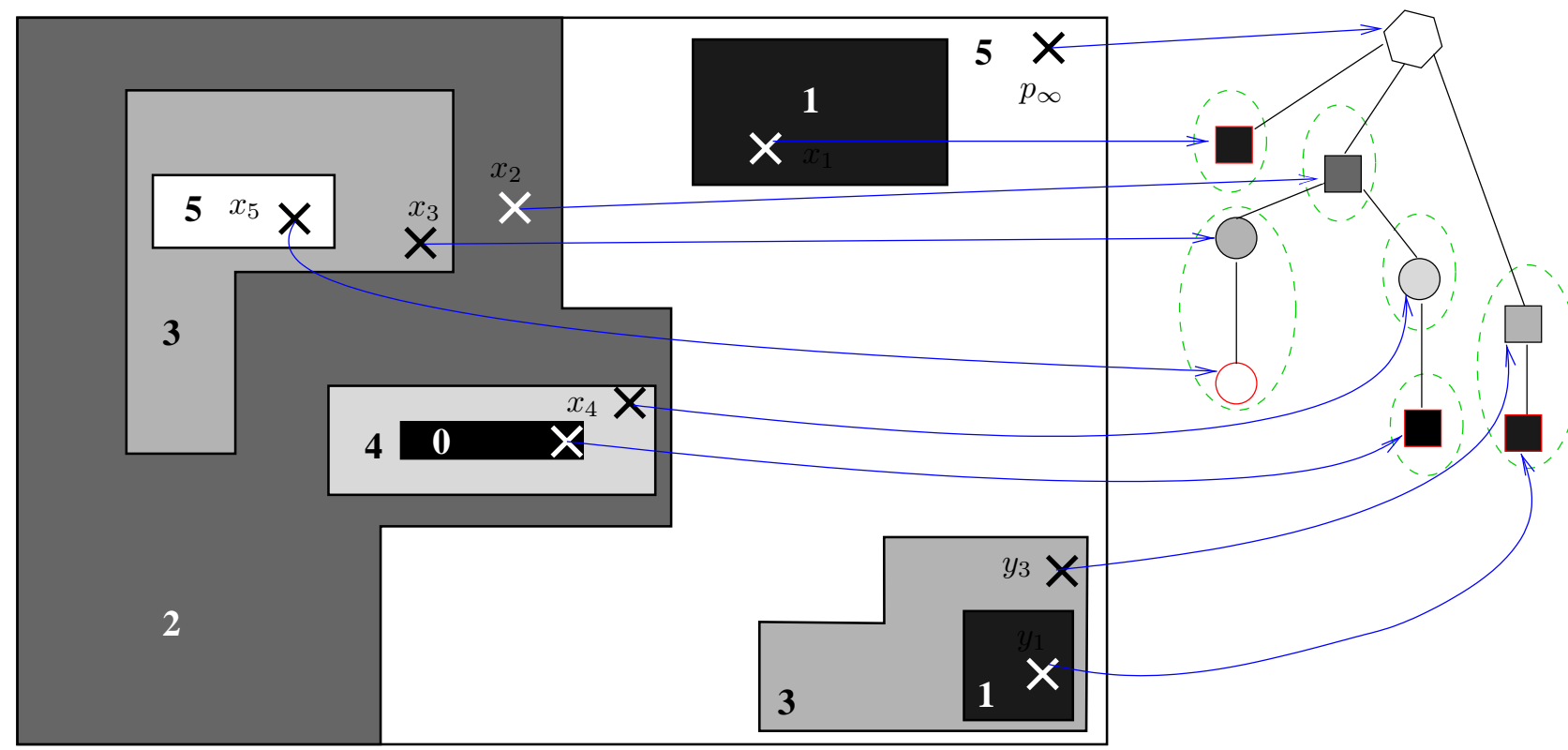

Figure 1. Example of tree associated to an image. Left: the sample image. Right: associated tree. Squares represent shapes of inferior type, while circles represent shapes of superior type. The maximal monotone sections are indicated by ellipses. Various points $x$ are marked in the image, and, for each of them, a line points to the smallest limit shape $S_{x}$ associated to it. A red border denotes a leaf.

By the first part of the proof, for any $Q \in[R, B]$, either $T \subseteq Q$ or $Q \subseteq T$. Then, either there is $Q \in[R, B]$ such that $T \subseteq Q$, or $Q \subseteq T$, for all $Q \in[R, B]$. In the first case, we have that $T \subseteq \sup [R, B]=S$, in the second, $S=\sup [A, B] \subseteq T$.

Proposition 3.11. Let $A_{1}, A_{2} \neq \emptyset$, and $\left[A_{1}, B_{1}\right],\left[A_{2}, B_{2}\right]$ two branches such that

$$
\left[A_{1}, B_{1}\right] \cap\left[A_{2}, B_{2}\right] \neq \emptyset .
$$

Without loss of generality we may assume that $A_{1}, A_{2}, B_{1}, B_{2}$ are limit shapes. Then $\left[A_{1} \cap A_{2}, B_{1} \cup B_{2}\right]$ is a branch.

Proof. Let $S \in \mathcal{S}(u)$ be such that $A_{1} \subseteq S \subseteq B_{1}$ and $A_{2} \subseteq S \subseteq B_{2}$. Thus $B_{1} \cap B_{2} \neq \emptyset$, and, therefore, $B_{1}$ and $B_{2}$ are nested. The limit shapes $A_{1}$ and $A_{2}$ are also nested. Indeed, we observe that $A_{2}=\inf \left[A_{2}, S\right]$. For any $R \in\left[A_{2}, S\right], R \subseteq B_{1}$ and since there is no bifurcation in $\left[A_{1}, B_{1}\right], A_{1} \cap R \neq \emptyset$. If there is $R \in\left[A_{2}, S\right]$ such that $R \subseteq A_{1}$, then $A_{2}=\inf \left[A_{2}, S\right] \subseteq A_{1}$. If this does not happen, $A_{1} \subseteq R$ for all $R \in\left[A_{2}, S\right]$, and we have that $A_{1} \subseteq \inf \left[A_{2}, S\right]=A_{2}$.

By symmetry, we may assume that either

$$
\begin{aligned}
& A_{1} \subseteq B_{1} \subseteq A_{2} \subseteq B_{2}, \text { or } \\
& A_{1} \subseteq A_{2} \subseteq B_{1} \subseteq B_{2}, \text { or } \\
& A_{1} \subseteq A_{2} \subseteq B_{2} \subseteq B_{1} .
\end{aligned}
$$

If $(i)$ holds, then

$$
A_{1} \subseteq S \subseteq B_{1} \subseteq A_{2} \subseteq S \subseteq B_{2}
$$


and $B_{1}=A_{2}=S$. This case can be subsumed under the case (ii). If (iii) holds, then $\left[A_{1} \cap A_{2}, B_{1} \cup B_{2}\right]=\left[A_{1}, B_{1}\right]$ is a branch. Thus, we may assume that $(i i)$ holds. We prove that there is no bifurcation in $\left[A_{1}, B_{2}\right]$.

Let $R$ a shape such that $R \subseteq B_{2}$. Since $\left[A_{2}, B_{2}\right]$ is a branch, $R \cap A_{2} \neq \emptyset$. By Proposition 3.10, either $A_{2} \subseteq R$ or $R \subseteq A_{2}$. In the first case, $A_{1} \subseteq R$, while, in the second case, $R \subseteq B_{1}$ and, since $\left[A_{1}, B_{1}\right]$ is a branch, $R \cap A_{1} \neq \emptyset$. This holds for any $R \subseteq B_{2}$, proving that $\left[A_{1}, B_{2}\right]$ is a branch.

Proposition 3.12. Let $A_{1}, A_{2} \neq \emptyset$, and let $\left[A_{1}, B_{1}\right],\left[A_{2}, B_{2}\right]$ be two monotone sections of the same type such that $\left[A_{1}, B_{1}\right] \cap\left[A_{2}, B_{2}\right] \neq \emptyset$. Without loss of generality we may assume that $A_{1}, A_{2}, B_{1}, B_{2}$ are limit shapes. Then $\left[A_{1} \cap A_{2}, B_{1} \cup B_{2}\right]$ is a monotone section.

Proof. By Proposition 3.11, we know that $\left[A_{1} \cap A_{2}, B_{1} \cup B_{2}\right]$ is a branch. As in the proof of Proposition 3.11, we may reduce the study to one of the three cases $(i),(i i)$ or $(i i i)$. In case (iii), the conclusion is immediate. As we observed above, $(i)$ can be subsumed under case (ii). Thus, assume that (ii) holds. Assume that the type of both intervals is the superior one. Let $R \in\left[A_{1}, B_{2}\right]$. Since $R \cap A_{2} \neq \emptyset$, then either $A_{2} \subseteq R$, or $R \subseteq A_{2}$. In the first case, $R \in\left[A_{2}, B_{2}\right]$, in the second, $R \in\left[A_{1}, B_{1}\right]$. In any case, $R$ is a shape of superior type. Thus $\left[A_{1}, B_{2}\right]$ is an interval with all shapes of superior type. The proof is similar if both intervals are of inferior type.

Propositions 3.11 and 3.12 permit us to define the maximal branch and maximal monotone section containing a given shape $S \in \mathcal{S}(u)$. Indeed, we define $\mathcal{B}(S)$, the maximal branch containing $S$, as

$$
\mathcal{B}(S)=\cup\{[A, B]:[A, B] \text { is a branch s.t. } S \in[A, B]\} .
$$

We define $\mathcal{M}(S)$, the maximal monotone section containing $S$, as

$$
\mathcal{M}(S)=\cup\{[A, B]:[A, B] \text { is a monotone section s.t. } S \in[A, B]\} .
$$

We observe that

$$
\mathcal{B}(S)=[\inf \mathcal{B}(S), \sup \mathcal{B}(S)], \text { and } \mathcal{M}(S)=[\inf \mathcal{M}(S), \sup \mathcal{M}(S)]
$$

As a consequence of Proposition 3.11 we obtain the following corollary:

Corollary 3.13. Let $x \in \bar{\Omega}$. The upper branch at $x, \mathcal{B}_{x}$, is a branch.

Proposition 3.14. Any two different leaves in the tree of shapes of $u$ are disjoint.

Proof. Let $L_{1}, L_{2}$ be two different leaves. Assume that $L_{1}=\inf \left[A_{1}, B_{1}\right], L_{2}=\inf \left[A_{2}, B_{2}\right]$. If they are not disjoint, then either $L_{1} \subseteq L_{2}$ or $L_{2} \subseteq L_{1}$. By symmetry, we may assume that $L_{1} \subseteq L_{2}$. Then, for any $S \in\left[A_{1}, B_{1}\right]$ and any $T \in\left[A_{2}, B_{2}\right]$, we have that $S \cap T \subseteq L_{1} \neq \emptyset$. Thus, $S$ and $T$ are nested. Thus either

$$
\begin{aligned}
\exists S & \in\left[A_{1}, B_{1}\right] \text { s.t. } \forall T \in\left[A_{2}, B_{2}\right], S \subseteq T, \text { or } \\
\forall S \in\left[A_{1}, B_{1}\right], & \exists T \in\left[A_{2}, B_{2}\right] \text { s.t. } T \subseteq S .
\end{aligned}
$$

If $(*)$ holds, then $S \subseteq L_{2}$. If $S \neq L_{2}$, then $L_{2}$ would not be a leaf. Thus, $S=L_{2}$. Since $L_{1}=\inf \left[A_{1}, S\right]$, if $R \in\left[A_{1}, S\right], R \subseteq L_{2}$. If, for some $R \in\left[A_{1}, S\right], R \neq L_{2}$, then $L_{2}$ would not be a leaf. Thus, for any $R \in\left[A_{1}, S\right]$, $R=L_{2}$. Therefore $L_{1}=L_{2}$, a contradiction. If $(* *)$ holds, then $L_{2} \subseteq L_{1}$. Hence, $L_{1}=L_{2}$. This contradiction proves that $L_{1}$ and $L_{2}$ must be disjoint.

\section{Reconstruction of the image from its tree}

This section explains how an image can be reconstructed from its tree; more precisely, we prove that the image is perfectly defined by its shapes and the levels at which they are extracted. 
Definition 4.1. We call lower (resp. upper) level shape at level $\lambda$ at $x \in \bar{\Omega}$ a pair $(\lambda, S=\operatorname{Sat}(\operatorname{cc}([u<\lambda])))$ (resp. $(\lambda, S=\operatorname{Sat}(\operatorname{cc}([u \geq \lambda]))))$ such that $x \in S$. We denote their family by $G_{\lambda, x}$ (resp. $\left.F_{\lambda, x}\right)$. We also denote

$$
\mathrm{LS}_{x}=G_{\lambda, x} \cup F_{\lambda, x}
$$

The relation $(\lambda, S) \preccurlyeq(\mu, T)$, defined as $S \subsetneq T$ or $\left(S=T\right.$ and $\lambda \leq \mu$, if $(\lambda, S) \in G_{\lambda, x},(\mu, T) \in G_{\mu, x} ;$ resp. $\lambda \geq \mu$, if $\left.(\lambda, S) \in F_{\lambda, x},(\mu, T) \in F_{\mu, x}\right)$, is a total order relation in $\operatorname{LS}_{x}$, provided that $S \neq \bar{\Omega} \neq T$.

Lemma 4.2. Let $x \in \bar{\Omega}, A$ be a connected component of a level set of $u$, and $(\lambda, \operatorname{Sat}(A)) \in \operatorname{LS}_{x}$. If $x \notin A$, then there exists $(\lambda, \operatorname{Sat}(B)) \in \operatorname{LS}_{x}$ such that $\operatorname{Sat}(B) \subsetneq \operatorname{Sat}(A)$ and $B$ is of a different type than $A$.

Proof. Let $T$ be the hole of $A$ containing $x$. If $A=\operatorname{cc}([u<\lambda])$, then $\partial T \subseteq[u \geq \lambda]$, and, since $\partial T$ is connected, we have that $(\lambda, \operatorname{Sat}(T)=T) \in F_{\lambda, x}$, with $T \subsetneq \operatorname{Sat}(A)$. Thus, we may assume that $A=\operatorname{cc}([u \geq \lambda])$.

Let $H$ be the family of connected components of $[u<\lambda]$ inside $T$. Since $H$ is a family of open and disjoint sets, we can enumerate its elements, $H_{1}, \ldots, H_{n} \ldots$ Let $D_{n}=\bar{T} \backslash \bigcup_{i=1}^{n} \operatorname{Sat}\left(H_{i}\right)$. Clearly, $D_{n}$ is closed. Let us prove that $D_{n}$ is connected. Let $G \neq \emptyset$ be an open and closed subset of $D_{n}$. Now, it is easy to check that

$$
\partial D_{n}=\partial T \cup \bigcup_{i=1}^{n} \partial \operatorname{Sat}\left(H_{i}\right)
$$

and we observe that each term of this union is connected. Hence, if $G$ meets one of $\partial \operatorname{Sat}\left(H_{i}\right)$, it contains it. Let $G^{\prime}$ be the union of $G$ and the sets $\operatorname{Sat}\left(H_{i}\right)$ whose boundary is contained in $G$. Obviously $G^{\prime}$ is open in $\bar{T}$. We observe that, if the boundary of $\operatorname{Sat}\left(H_{i}\right)$ is contained in $G$, then $G \cup \operatorname{Sat}\left(H_{i}\right)=G \cup \overline{\operatorname{Sat}\left(H_{i}\right)}$, and, therefore, $G^{\prime}$ is also closed in $\bar{T}$. Due to the connectedness of $\bar{T}, G^{\prime}=\bar{T}$ and, therefore, $G=D_{n}$. This proves that $D_{n}$ is connected.

If the number of $H_{i}$ is finite, this proves that $\bar{T} \backslash \bigcup_{i} \operatorname{Sat}\left(H_{i}\right)$ is connected. If they are infinite, the sets $D_{n}$ form a decreasing sequence of continua, thus, their intersection is a continuum, thanks to Zoretti's theorem.

Since $\partial T \subseteq A$ and is connected, we have that $\bar{T} \backslash \bigcup_{i} \operatorname{Sat}\left(H_{i}\right) \subseteq A$. Now, since $x \notin A$, it follows that $x \in \bigcup_{i} \operatorname{Sat}\left(H_{i}\right)$, which implies the conclusion of the lemma.

\subsection{Direct reconstruction}

Theorem 4.3. Let $x \in \bar{\Omega}$. If $S_{x} \neq \bar{\Omega}$, then

$$
u(x)=\lim _{\operatorname{LS}_{x} \ni(\lambda, S) \searrow} \lambda .
$$

If $S_{x}=\bar{\Omega}$, then

$$
u(x)=\inf _{(\lambda, \bar{\Omega}) \in G_{\lambda, x}} \lambda=\max _{(\lambda, \bar{\Omega}) \in F_{\lambda, x}} \lambda .
$$

Equation (3) deserves some explanation. It must be interpreted as

$$
\forall \varepsilon>0, \exists(\lambda, S) \in \mathrm{LS}_{x}, \forall(\mu, T) \preccurlyeq(\lambda, S),|u(x)-\mu| \leq \varepsilon .
$$

Loosely speaking, $u(x)$ is the level of the smallest shape containing $x$. But this "smallest shape" does not necessarily exist, and, moreover, the relation $\preccurlyeq$ takes into account that the same shape can sometimes be extracted at several levels. 
Proof. Suppose first that $S_{x}=\bar{\Omega}$. Let $\lambda \leq u(x)$ and $C_{1}=\operatorname{cc}([u \geq \lambda], x)$. Then $\operatorname{Sat} C_{1}=\bar{\Omega}$. Now, if $C_{2}=\operatorname{cc}([u<\lambda])$, is such that $C_{2} \cap C_{1}=\emptyset$, we have that $\operatorname{Sat}\left(C_{2}\right) \neq \bar{\Omega}$, and, therefore, Sat $C_{2}$ does not contain $x$. This proves that

$$
u(x) \leq \inf _{(\lambda, \bar{\Omega}) \in G_{\lambda, x}} \lambda .
$$

Now, if $\lambda_{n}=u(x)+1 / n$, it is clear that $\left(\lambda_{n}, \bar{\Omega}\right) \in G_{\lambda, x}$, thus,

$$
\inf _{(\lambda, \bar{\Omega}) \in G_{\lambda, x}} \lambda \leq \lambda_{n}
$$

which gives the equality with $u(x)$ as $n$ tends to infinity. The second equality follows by using the same argument, the maximum being reached this time since $(u(x), \bar{\Omega}) \in F_{\lambda, x}$.

We assume now that $S_{x} \subsetneq \bar{\Omega}$. There is some shape $S^{*} \varsubsetneqq \bar{\Omega}$, containing $S_{x}$, and let $\lambda^{*}$ be its associated level. Let us consider the shape $S_{0}=\operatorname{Sat}(\operatorname{cc}([u \geq u(x)], x))$. It is clear that $\left(u(x), S_{0}\right) \in \operatorname{LS}_{x}$. Let $(\lambda, S) \in \operatorname{LS}_{x}$ be such that $(\lambda, S) \preccurlyeq\left(u(x), S_{0}\right)$ if $S_{0} \varsubsetneqq \bar{\Omega}$, or $S \varsubsetneqq \bar{\Omega}$ if $S_{0}=\bar{\Omega}$. We claim that $\lambda \geq u(x)$, this inequality being strict if $S_{0}=\bar{\Omega}$.

If $S$ is a lower shape, we have that $S \varsubsetneqq S_{0}$. Since $S=\operatorname{Sat}(\operatorname{cc}([u<\lambda]))$, if we have that $\lambda \leq u(x)$, we deduce that this $\operatorname{cc}([u<\lambda])$ does not contain $x$, and, thus, that $x$ is in one of its internal holes, as it is $\operatorname{cc}([u \geq u(x)], x)$ and, therefore, also its saturation, which is impossible. Thus, we must have that $\lambda>u(x)$.

If $S$ is an upper shape, we write $S=\operatorname{Sat}(\operatorname{cc}([u \geq \lambda]))$. If $x \in \operatorname{cc}[u \geq \lambda]$, we have that $u(x) \geq \lambda$, in which case $\operatorname{cc}([u \geq \lambda]) \supseteq \operatorname{cc}([u \geq u(x)], x)$. It follows that $S_{0} \neq \bar{\Omega}$ and $\operatorname{cc}([u \geq \lambda]=\operatorname{cc}([u \geq u(x)], x)$. Then the order relation yields that $\lambda \geq u(x)$, and, thus, we have the equality. Thus, we may assume that $x$ is in an internal hole of $\operatorname{cc}([u \geq \lambda])$. Since $\operatorname{cc}([u \geq u(x), x)$ is not included in this hole, it must intersect $\operatorname{cc}([u \geq \lambda])$, and, hence, it contains it (strictly). This implies that $\lambda>u(x)$.

For $\varepsilon>0$, let us consider the shape $S_{\varepsilon}=\operatorname{Sat}(\operatorname{cc}([u<u(x)+\varepsilon], x))$ and assume that $S_{\varepsilon} \neq \bar{\Omega}$. Let $(\lambda, S) \in \operatorname{LS}_{x}$ be such that $(\lambda, S) \preccurlyeq\left(u(x)+\varepsilon, S_{\varepsilon}\right)$.

Suppose that $S$ is an upper shape, $S=\operatorname{cc}([u \geq \lambda]) \neq \bar{\Omega}$. If $\lambda \geq u(x)+\epsilon$, then $x \notin \operatorname{cc}([u \geq \lambda])$ and there is an internal hole $H$ of this set containing $x$. This implies that $\operatorname{cc}([u<u(x)+\varepsilon], x)$ is also contained in $H$, and since, by assumption, $S \neq \bar{\Omega}$, we have that $\operatorname{Sat}(H)=H$. It follows that $S_{\varepsilon} \subseteq H \varsubsetneqq S$, which is contrary to the assumption that $S \subseteq S_{\varepsilon}$. Thus, $\lambda<u(x)+\varepsilon$.

If $S=\operatorname{Sat}(\operatorname{cc}([u<\lambda]))$ is a lower shape, and $S=S_{\varepsilon}$, we must have that $\lambda \leq u(x)+\varepsilon$. In case $S \mp S_{\varepsilon}$, we have either $x \in \operatorname{cc}([u<\lambda])$, in which case $\lambda<u(x)+\epsilon$, or $x$ is in an internal hole $H$ of $\operatorname{cc}([u<\lambda])$. In the second case, since $\operatorname{cc}([u<u(x)+\varepsilon], x) \nsubseteq \subseteq H$, we get $\operatorname{cc}([u<u(x)+\varepsilon], x) \cap \operatorname{cc}([u<\lambda]) \neq \emptyset$, which, in turn, implies that $\operatorname{cc}([u<\lambda] \subseteq \operatorname{cc}([u<u(x)+\varepsilon], x)$ and, thus, $\lambda \leq u(x)+\varepsilon$. The above results allow to conclude in the two possible configurations: $S_{0}=\bar{\Omega}$ or $S_{0} \varsubsetneqq \bar{\Omega}$.

If $S_{0}=\bar{\Omega}$, we have that $S^{*} \varsubsetneqq \bar{\Omega}$, implying (see above) that $\lambda^{*}>u(x)$ and we easily derive

$$
\left(\lambda^{*}, S_{\lambda^{*}-u(x)}\right) \preccurlyeq\left(\lambda^{*}, S^{*}\right) .
$$

Let $\varepsilon>0$, and let $\varepsilon^{\prime}=\min \left(\varepsilon, \lambda^{*}-u(x)\right)$. Since $S_{\varepsilon^{\prime}} \varsubsetneqq \bar{\Omega}$, if $(\lambda, S) \preccurlyeq\left(u(x)+\varepsilon^{\prime}, S_{\varepsilon^{\prime}}\right)$, the above results prove that $\lambda \leq u(x)+\varepsilon^{\prime}$, and, since $S \mp \bar{\Omega}$, we have that $\lambda>u(x)$. Therefore $|\lambda-u(x)| \leq \varepsilon^{\prime} \leq \varepsilon$, proving equation (3).

Now, we assume that $S_{0} \varsubsetneqq \bar{\Omega}$. If, for some $\varepsilon>0$, we have that $S_{\varepsilon} \varsubsetneqq \bar{\Omega}$, we conclude in the same manner as in the previous case. Otherwise, let $(\lambda, S) \preccurlyeq\left(u(x), S_{0}\right)$. Observe that $\lambda \geq u(x)$. If $S=\operatorname{Sat}(\operatorname{cc}([u<\lambda]))$ is of lower type, necessarily $\lambda=u(x)$ and $x$ is in a hole $H$ of $\operatorname{cc}([u<\lambda])$. Therefore $\operatorname{cc}([u \geq u(x)], x) \subseteq H \varsubsetneqq S$, which contradicts $(\lambda, S) \preccurlyeq\left(u(x), S_{0}\right)$. Then $S=\operatorname{Sat}(C)$ is of upper type. If $\lambda>u(x)$, then $x$ is in a hole $H$ of $C$, and, thanks to Lemma 4.2, there is some lower shape $S^{\prime}$ at level $\lambda$ contained in $H$ and containing $x$. This easily implies that $S_{\lambda-u(x)} \subseteq S^{\prime} \varsubsetneqq \bar{\Omega}$, contradicting our previous assumption. In conclusion, $(\lambda, S) \preccurlyeq\left(u(x), S_{0}\right)$ implies that $\lambda=u(x)$ (and $S=S_{0}$ ), proving equation (3), the infimum being actually reached at $\left(u(x), S_{0}\right)$. 


\subsection{Indirect reconstruction}

The formulae for reconstruction we have discussed above are complex in the sense that they involve a limit. We provide here a simpler, algebraic reconstruction, in two steps: first, the level sets are deduced from the tree, then the reconstruction of the image is straightforward, given by

$$
u(x)=\sup \{\lambda, x \in[u \geq \lambda]\}=\inf \{\lambda, x \in[u<\lambda]\}
$$

The reconstruction will derive from the following lemma:

Lemma 4.4. Let $x \in \bar{\Omega}$ and $\lambda \in \mathbb{R}$ be such that $\operatorname{LS}_{\lambda, x} \neq \emptyset$. Then

$$
\bigcap_{A \in \mathrm{LS}_{\lambda, x}} A \in \mathrm{LS}_{\lambda, x}
$$

Proof. If there exists $G \in G_{\lambda, x}$ such that $G \subseteq A$ for all $A \in F_{\lambda, x}$, then $G=\bigcap_{A \in \mathrm{LS} \lambda, x} A$. Indeed, obviously, we have that $G \subseteq \bigcap_{A \in F_{\lambda, x}} A$. Thus, it will be sufficient to prove that $G \subseteq \bigcap_{A \in G_{\lambda, x}} A$. For that, assume, on the contrary, that there is some $G_{1} \in G_{\lambda, x}$ such that $G$ is not contained in $G_{1}$. Since both shapes intersect (at $x$ ), we must have that $G_{1} \varsubsetneqq G$, hence $x$ cannot be in the connected component of $[u<\lambda]$ defining $G$. Then, by Lemma 4.2, we find $F \in F_{\lambda, x}$ such that $F \varsubsetneqq G$, a contradiction to our assumption. Thus, we may assume that

$$
\forall G \in G_{\lambda, x}, \exists F \in F_{\lambda, x}, F \subseteq G
$$

Note that $(*)$ implies that

$$
\bigcap_{A \in \mathrm{LS}_{\lambda, x}} A=\bigcap_{F \in F_{\lambda, x}} F .
$$

Since elements of $F_{\lambda, x}$ are closed, by Lindelöf's theorem, their intersection can be written as the intersection of a sequence of these sets, and, since they are continua, their common part $K$ is a continuum, thanks to Zoretti's theorem. Then $\bar{\Omega} \backslash K$ can be written as

$$
\bar{\Omega} \backslash K=\bigcup_{F \in F_{\lambda, x}}(\bar{\Omega} \backslash F)
$$

For such an $F, \bar{\Omega} \backslash F$ is connected. Hence $\bar{\Omega} \backslash K$ is a union of connected sets meeting a connected set $\bar{\Omega} \backslash F_{0}$ $\left(F_{0}\right.$ being an arbitrary element of $F_{\lambda, x}$ ), thus, it is connected. Due to unicoherency, $\partial K$ is thus connected.

Next, we observe that $\partial K \subseteq[u \geq \lambda]$. Indeed, let $y \in \partial K$ and $U$ be a connected neighborhood of $y$. There is some $z \in U \backslash K$, and, therefore, there is an $F \in F_{\lambda, x}$ such that $z \notin F$. Moreover $U \cap F \neq \emptyset$ and, since $U$ is connected, $U \cap \partial F \neq \emptyset$. Hence $U \cap[u \geq \lambda] \neq \emptyset$. Since this is valid for any connected neighborhood of $y$, we get

$$
y \in \overline{[u \geq \lambda]}=[u \geq \lambda]
$$

Therefore $\partial K$ is contained in a connected component of $[u \geq \lambda]$ and, since $K=\operatorname{Sat}(K)=\operatorname{Sat}(\partial K)$, we get

$$
K \subseteq \operatorname{Sat}(\operatorname{cc}([u \geq \lambda]))
$$

The opposite inclusion is then evident. 
Theorem 4.5. We have the following representation formulas

$$
\begin{aligned}
& {[u \geq \lambda] \cap\left\{x, S_{x} \neq \bar{\Omega}\right\}=\left\{x \in \bar{\Omega}, \bigcap_{A \in \mathrm{LS}_{\lambda, x}} A \in F_{\lambda, x}\right\}=\bigcup_{x, S_{x} \neq \bar{\Omega}} \bigcup_{F \in F_{\lambda, x}}\left(F \backslash \underset{G \in G_{\lambda, x}, G \subseteq F}{\bigcup} G\right), \text { and }} \\
& {[u<\lambda] \cap\left\{x, S_{x} \neq \bar{\Omega}\right\}=\left\{x \in \bar{\Omega}, \bigcap_{A \in \mathrm{LS}_{\lambda, x}} A \in G_{\lambda, x}\right\}=\bigcup_{x, S_{x} \neq \bar{\Omega}} \bigcup_{G \in G_{\lambda, x}}\left(G \backslash \underset{F \in F_{\lambda, x}, F \subseteq G}{\bigcup} F\right) .}
\end{aligned}
$$

Proof. Let $x$ be such that $u(x) \geq \lambda$, and $F=\operatorname{Sat}(\operatorname{cc}([u \geq \lambda], x))$. We prove that $F=\bigcap_{A \in \operatorname{LS}_{\lambda, x}} A$. Indeed, if $G=\operatorname{Sat}(\operatorname{cc}([u<\lambda])) \in G_{\lambda, x}$, then $x$ is in an internal hole of $\operatorname{cc}([u<\lambda])$, and so it is $\operatorname{cc}([u \geq \lambda], x)$. Thus $F \subseteq G$. Similarly, if $F^{\prime} \in F_{\lambda, x}$ is such that $F^{\prime} \subsetneq F$, thanks to Lemma 4.2, there is some $G \in G_{\lambda, x}$ such that $F^{\prime} \subsetneq G \subsetneq F$, which was shown to be impossible.

Conversely, let $x \in \bar{\Omega}$ be such that $F=\bigcap_{A \in \mathrm{LS}_{\lambda, x}} A \in F_{\lambda, x}$. We write $F=\operatorname{Sat}(\operatorname{cc}([u \geq \lambda]))$. If $x$ were in an internal hole of $\operatorname{cc}([u \geq \lambda])$, thanks to Lemma 4.2 , there would be some $G \in G_{\lambda, x}$ such that $G \subsetneq F$, contradicting the minimality of $F$. Thus $x \in \operatorname{cc}([u \geq \lambda])$.

We have proved the first equality in the first representation formula. The other equalities are direct consequences of this one.

\section{Finiteness of the tree For images With GRAins of Minimal POSitive Size}

Definition 5.1. We say that an image $u$ has grains of minimal positive size if there exists some $\delta>0$ such that for any connected component $K$ of an upper or lower level set of $u,|K| \geq \delta$.

After application of an area opening and an area closing filters of area $\delta$ (see $[51,52]$ ), any image verifies this property (see [6]). Provided such an image, we will show that the tree, whereas it can have an infinite number of nodes, actually has a finite structure. In this whole section, we will suppose that $u$ is an image of minimal positive size $\delta$.

Lemma 5.2. For each $\lambda \in \mathbb{R}$ there is a finite number of connected components of $[u \geq \lambda]$ and each component has a finite number of holes.

Proof. Let $\lambda \in \mathbb{R}$. Since each connected component of $[u \geq \lambda]$ has area $\geq \delta$, there must be a finite number of them. Let $Y$ be a component of $[u \geq \lambda]$ and let $H$ be a hole of $Y$. Since $H$ is open, $H$ cannot be covered by components of $[u \geq \lambda]$. Hence $H \cap[u<\lambda] \neq \emptyset$. We conclude that each hole of $Y$ contains a component of $[u<\lambda]$. Hence there may be only a finite number of them.

Lemma 5.3. (i) Let $T$ be any shape. Then $|T| \geq \delta$.

(ii) Let $S \subseteq T$ be two shapes of different type. Then $|T \backslash S| \geq \delta$.

Proof. (i) Since any shape $T$ contains a connected component of an upper or lower level set, then $|T| \geq \delta$.

(ii) Suppose that $S=\operatorname{Sat}(X), T=\operatorname{Sat}(Y)$, where $X$ and $Y$ are connected components of level sets of $u$. If $X \cap Y=\emptyset$, then $Y \subseteq T \backslash S$, thus, $|T \backslash S| \geq|Y| \geq \delta$. Therefore, we may assume that $X \cap Y \neq \emptyset$.

Suppose first that $X=\operatorname{cc}([u \geq \lambda])$ and $Y=\operatorname{cc}([u<\mu]), \lambda, \mu \in \mathbb{R}$. As $X \cap Y \neq \emptyset, \lambda<\mu$. Assume that $Y \backslash S \subseteq[u \geq \lambda]$. In this case, we could write

$$
Y=(Y \cap S) \cup(Y \cap([u \geq \lambda] \backslash S))
$$

Now, $S$ being closed, $Y \cap S$ is a closed set of $Y$, and $[u \geq \lambda] \backslash S$, being a finite union of connected components of $[u \geq \lambda]$, is also closed. Hence, we have a partition of the set $Y$ into two closed subsets, contradicting its 
connectedness. Therefore, $Y \cap([u<\lambda] \backslash S) \neq \emptyset$ In particular, $Y \backslash S$ contains a connected component of $[u<\lambda]$, hence, we have that

$$
|T \backslash S| \geq|Y \backslash S| \geq \delta
$$

In the same way, if $X=\operatorname{cc}([u<\lambda])$ and $Y=\operatorname{cc}([u \geq \mu])$, we have that $\mu<\lambda$, and, since $Y$ is connected and meets both $S$ and $\bar{\Omega} \backslash S$, we also have that $Y \cap \partial S \neq \emptyset$. Since $\partial S \subseteq \partial X \subseteq[u \geq \lambda]$ and $S \cap \partial S=\emptyset$, we have that $(Y \backslash S) \cap[u \geq \lambda] \neq \emptyset$, and, therefore, $Y \backslash S$ contains a connected component of $[u \geq \lambda]$, yielding $(*)$.

Proposition 5.4. There is a finite number of leaves in the tree of shapes of $u$.

Proof. Since any leaf $T$ is an intersection of a nested family of shapes, which, by Lemma 5.3( $i i)$, are all of measure $\geq \delta$, then $|T| \geq \delta$. Since, by Proposition 3.14, any two different leaves are disjoint, we conclude that there must be a finite number of them.

Theorem 5.5. There is a finite number of maximal monotone sections in the tree of shapes of $u$.

Proof. Suppose that there is an infinite number of maximal monotone sections $\left[S_{n}^{*}, T_{n}^{*}\right]$ in the tree of shapes of $u$. Without loss of generality, we may assume that all are of the same type. Then $\left[S_{i}^{*}, T_{i}^{*}\right] \cap\left[S_{j}^{*}, T_{j}^{*}\right]=\emptyset$, for all $i \neq j$. Let us consider $S_{i}$ shapes such that $S_{i} \in\left[S_{i}^{*}, T_{i}^{*}\right]$ for all $i \geq 1$. If there is an infinite set of indices $I \subseteq \mathbb{N}$ such that $S_{i} \cap S_{j}=\emptyset$ for all $i, j \in I$, then by Lemma $5.3(i), \bar{\Omega}$ would be of infinite measure. Thus there is only a finite number of indices $i \in \mathbb{N}$ such that the $S_{i}$ are two by two disjoint. Thus there is $i_{1}$ such that $S_{i_{1}}$ contains an infinity of $S_{i}$. Without loss of generality, we may assume that $i_{1}=1$ and all $S_{i} \subseteq S_{1}$ for $i \geq 2$. By the same reasons there are at most a finite number of indices $i \geq 2$ such that $S_{i}$ are two by two disjoint. Thus, there is an index $i_{2}$, which we may assume to be $i_{2}=2$, such that $S_{2} \subseteq S_{1}$ and $S_{2}$ contains an infinity of $S_{i}$. In this way, we construct a family of sets $S_{n}$ such that $S_{n+1} \subseteq S_{n}$ for all $n \geq 1$.

Let us consider the interval $\left[S_{n+1}, S_{n}\right]$. We claim that $\left|S_{n} \backslash S_{n+1}\right| \geq \delta$. If there is a shape $S$ such that $S \subseteq S_{n}$ and $S \cap S_{n+1}=\emptyset$, then $S \subseteq S_{n} \backslash S_{n+1}$, and, by Lemma 5.3(i), $\left|S_{n} \backslash S_{n+1}\right| \geq \delta$. Thus, we may assume that there is no bifurcation in $\left[S_{n+1}, S_{n}\right]$. Suppose that all shapes in $\left[S_{n+1}, S_{n}\right]$ are of the same type. Thus, $\left[S_{n+1}, S_{n}\right]$ is a monotone section with all shapes of the same type. In this case, by Proposition $3.12,\left[S_{n+1}, S_{n}\right]$ would be a monotone section intersecting both $\left[S_{n+1}^{*}, T_{n+1}^{*}\right]$ and $\left[S_{n}^{*}, T_{n}^{*}\right]$, and there would exist a maximal monotone section containing both of them. This contradiction proves that there is a shape $Q_{n} \in\left[S_{n+1}, S_{n}\right]$ of different type as the ones of the maximal monotone sections that we are considering. By Lemma 5.3(ii), we have that $\left|S_{n} \backslash Q_{n}\right| \geq \delta$. Thus, also $\left|S_{n} \backslash S_{n+1}\right| \geq \delta$. Thus, $\left|S_{n}\right| S_{n+1} \mid \geq \delta$ for all $n \geq 1$. Now, since $\left(S_{n-1} \backslash S_{n}\right) \cap\left(S_{n} \backslash S_{n+1}\right)=\emptyset$ for all $n \geq 1$, this implies that $\bar{\Omega}$ must have infinite measure. This contradiction implies the statement of the theorem.

Corollary 5.6. There is a finite number of maximal branches in the tree of shapes of $u$.

Proof. Each maximal branch containing a maximal monotone section, their number cannot exceed the number of the maximal monotone sections, thus, it is finite.

\section{Application to the discrete case}

We switch now to the case of digital images, as they are used in computer science. Specifically, a digital image is an array of pixel values, $u_{i, j} \in \mathbb{R}, i=1, \ldots, W, j=1, \ldots, H$. To apply a theory valid for images to digital images is scarcely straightforward, and supposes some approximation. For example, in the digital case we have two notions of connectedness, 4-connectedness and 8-connectedness. In our case, we are able to interpret the discrete data in a continuous framework, so that previous results directly apply to the digital case. 

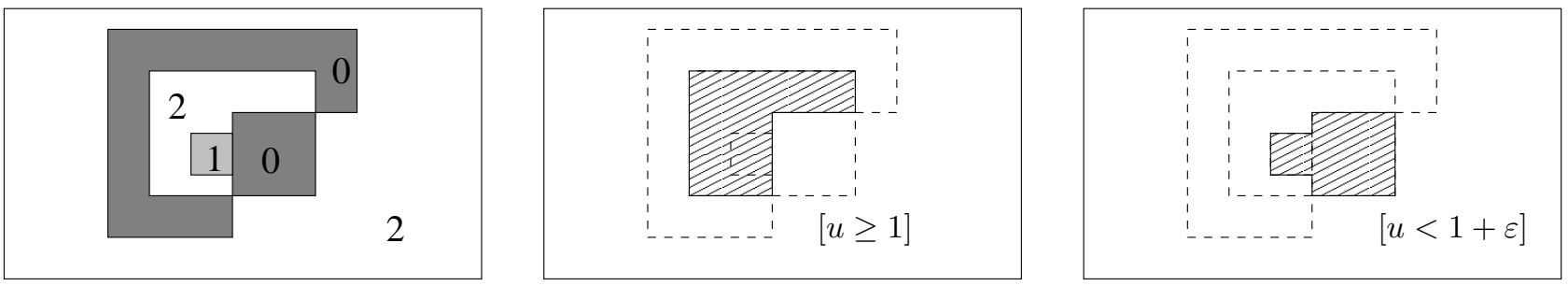

Figure 2. The usage of $M$-connectedness, or equivalently, the usage of 4-connectedness for upper and lower level sets contradicts Theorem 3.3. Left: the image $u_{c}$. Center: a connected component of the upper level set $\left[u_{c} \geq 1\right]$ with no internal hole. Right: idem for the connected component of the lower level set $\left[u_{c}<1+\varepsilon\right]$. These two shapes intersect and are not nested, even in the density sense (the measure of their symmetric difference is positive).

\subsection{Discrete tree}

For a set of real coordinates $(x, y) \in\left[\frac{1}{2}, W+\frac{1}{2}\right] \times\left[\frac{1}{2}, H+\frac{1}{2}\right]$, we define $\diamond(x, y)$ as the set of pairs of integral coordinates closest to $(x, y)$ :

$$
\diamond(x, y)=\left\{(i, j): \forall\left(i^{\prime}, j^{\prime}\right),\|(x-i, y-j)\| \leq\left\|\left(x-i^{\prime}, y-j^{\prime}\right)\right\|\right\} .
$$

We can see that $\diamond(x, y)$ has cardinal 1,2 or 4 . We define the image $u_{c}$ in the continuous framework associated to the digital image $u_{d}$ by

$$
\forall p \in \bar{\Omega}=\left[\frac{1}{2}, W+\frac{1}{2}\right] \times\left[\frac{1}{2}, H+\frac{1}{2}\right], \quad u_{c}(p)=\max _{(i, j) \in \triangleright p} u_{i, j} .
$$

The involved maximum makes $u_{c}$ an upper semicontinuous function and the image is constant at least on each unit open square centered at integral coordinates, which implies that $u_{c}$ has a minimal grain of size 1 . The above framework is therefore valid for $u_{c}$, and the tree of shapes of $u_{c}$ has a finite structure.

The consequence of (5) on discrete connectedness is the following: we must consider 8-connectedness for upper level sets of $u_{c}$, and 4-connectedness for lower level sets. In particular, we cannot adopt 4-connectedness for both, which is what $M$-connectedness, as defined in [3,5], assumes; a counterexample is given in Figure 2.

\subsection{Adaptive quantization}

The tree of shapes can be used to give schematic versions of an image. This can be done in two steps: first, remove small shapes of the tree, then select among the remaining maximal monotone sections only the most significant shapes. The filtered image is obtained by reconstruction of the resulting tree. The first step corresponds to the grain filter, as studied in the BV framework in [3], and is a generalization of the area opening and area closing, as introduced in $[51,52]$. The grain filter removes shapes of area smaller than a parameter $a$, whereas area opening (resp. closing) removes connected components of upper (resp. lower) level sets of area $\leq a$. The grain filter has the advantage over these filters to act simultaneously on upper and lower level sets. Moreover, it is selfdual on continuous functions as it was proved in [6] or in [26] for smooth functions of class $C^{N}, N$ being the dimension of the space. Thus this filter permits to simplify the topographic map of the image while keeping the geometric information of the image, given in terms of its "level lines" or its shapes, and do it in a symmetric way with respect to the upper and lower level sets, at least when the image is a continuous function. This prefiltering step is crucial in many image processing tasks, like morphological compression or registration, to mention two of them. The tree of shapes is well adapted to compute the action of the grain filter on an image in a simple and efficient way. 

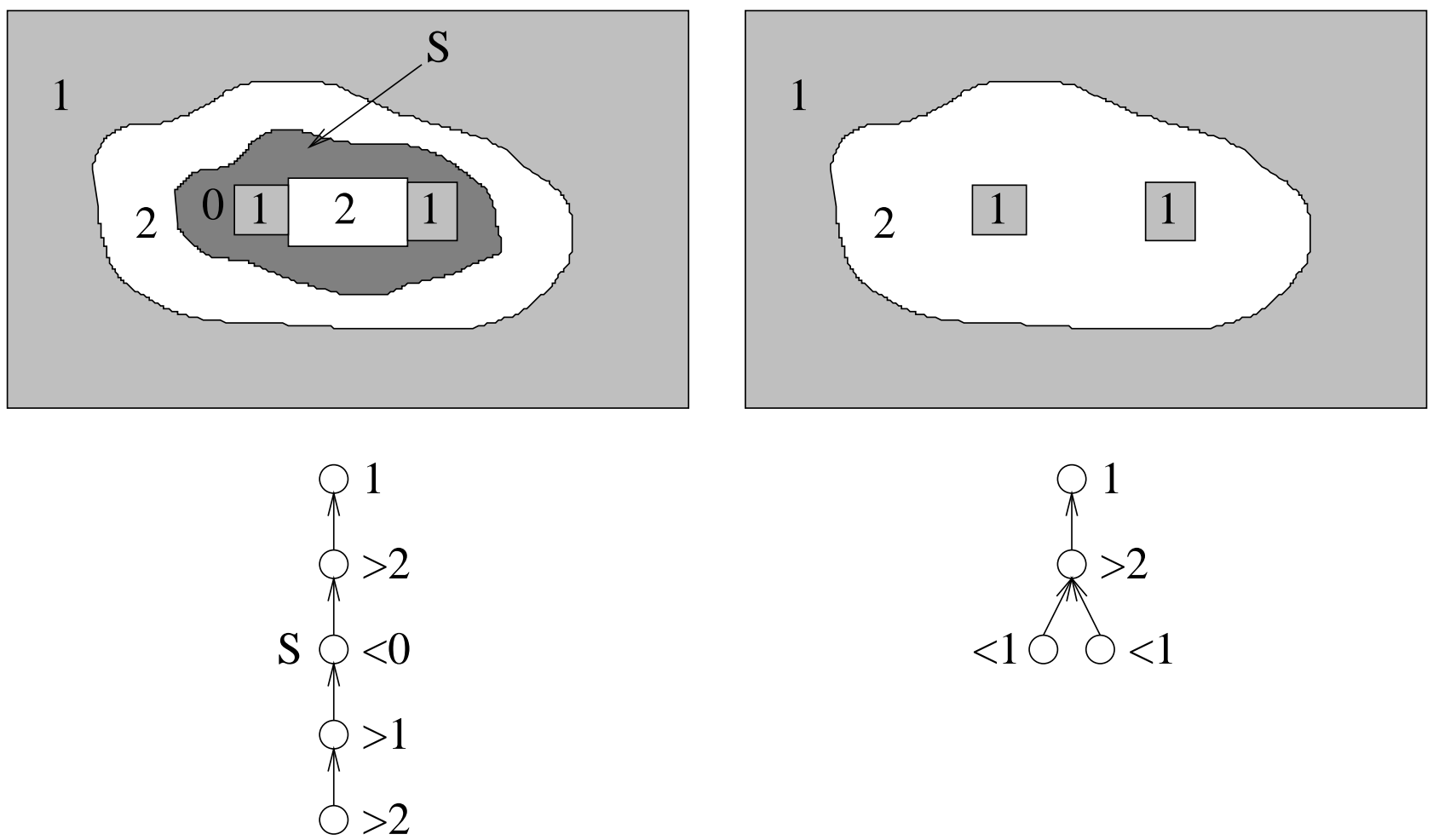

Figure 3. The removal of the shape $S$ should be prevented: the reconstructed image's tree structure is not the modified tree.

The second step works on maximal monotone sections: in each such section, we remove all shapes except the most significant one. The meaningfulness of a shape is chosen as the ratio $A / L^{2}$, where $L$ is the length of the boundary and $A$ the area. Thus the most round shapes remain. However, any other criterion that seems appropriate can be used, as for example the minimum gradient norm along the boundary. The remaining shape gets the mean gray level of the section, so as not to change the local contrast.

The idea behind this is twofold: small shapes represent only details of the image, or are mainly due to noise; the maximal monotone sections represent visual "objects" present multiple times in the image due to the gradation at their border, which comes from the smoothing during the acquisition process. Moreover, the removal of small shapes can eliminate some bifurcations, yielding longer maximal monotone sections.

However, we must be careful because some shapes cannot be removed without affecting the reconstruction. It can happen that the reconstructed image's tree of shapes is not the one on which the reconstruction was based. This is illustrated in Figure 3. To avoid this unfortunate result, it is sufficient to prevent the removal of the minimal shape of each maximal monotone section if one of its children is of a different type.

Figure 4 shows some results obtained by this adaptive quantization.

\subsection{Applications}

We review in this section some applications of the tree of shapes in digital image processing. In these applications the authors use the fast algorithm extracting the tree of shapes as presented in [35].

Desolneux et al. [12] use the tree of shapes to extract the level lines of the image with high contrast, in other words, the edges. Level lines having a minimal contrast above some threshold, which is based on statistical considerations, are considered meaningful. However, frequently, it happens that when a meaningful level line is detected, also are many other level lines inside the same monotone section. To get rid of this redundancy, they 


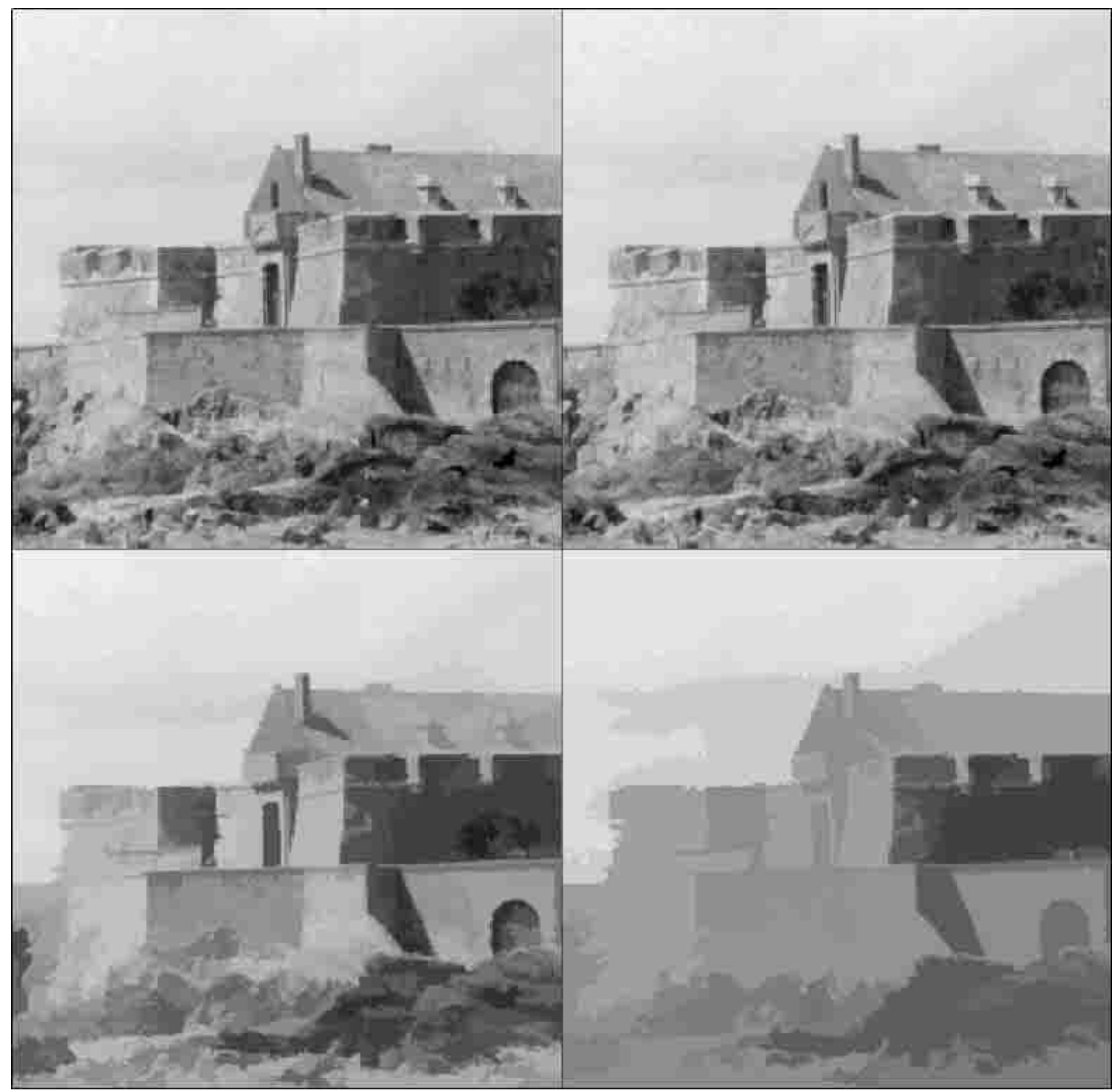

Figure 4. Effect of the adaptive quantization on an image of size $500 \times 500$. From left to right, top to bottom: $a=1$ (8,834 sections), $a=20(1,938), a=400$ (155) and $a=8,000$ (18).

keep only the most meaningful level lines in a monotone section. This simplification of the tree is very close to the adaptive quantization presented above, with the addition of a parameter measuring the meaningfulness.

Gousseau [21] uses the shapes to reproduce textures. Many shapes inside a texture are extracted and put in random locations in a blank image to synthesize the texture.

Dibos and Koepfler $[13,14]$ use the tree of shapes to construct a denoising filter which does not coincide with the grain filter [6]. Indeed, to decrease the total variation of an image by alteration of its level lines there 
are essentially two possibilities: either make them shorter, usually by evolution of a PDE, or to decrease their contrast. The latter solution is adopted in [13].

Shapes can also be used for registration purposes [15,34]: some invariant characteristics of shapes are extracted in different images and compared to get correspondences. Voting procedures can then be used to recover global displacement parameters. A more complex but more accurate method to extract the correspondences is proposed in [24], where pieces of level lines are compared.

\section{REFERENCES}

[1] L. Alvarez, F. Guichard, P.L. Lions and J.M. Morel, Axioms and fundamental equations of image processing. Arch. Rational Mech. Anal. 16 (1993) 200-257.

[2] L. Ambrosio, N. Fusco and D. Pallara, Functions of Bounded Variation and Free Discontinuity Problems. Oxford Mathematical Monographs (2000).

[3] L. Ambrosio, V. Caselles, S. Masnou and J.M. Morel, The Connected Components of Sets of Finite Perimeter. Eur. J. Math. 3 (2001) 39-92.

[4] C. Ballester, E. Cubero-Castan, M. Gonzalez and J.M. Morel, Image intersection and applications to satellite imaging, Preprint. C.M.L.A., École Normale Supérieure de Cachan (1998).

[5] C. Ballester and V. Caselles, The M-components of level sets of continuous functions in WBV. Publ. Mat. 45 (2001) $477-527$.

[6] V. Caselles and P. Monasse, Grain filters. J. Math. Imaging Vision (to appear).

[7] V. Caselles, B. Coll and J.M. Morel, Topographic Maps and Local Contrast Changes in Natural Images. Int. J. Comput. Vision 33 (1999) 5-27.

[8] V. Caselles, J.L. Lisani, J.M. Morel and G. Sapiro, Shape Preserving Histogram Modification. IEEE Trans. Image Process. 8 (1999).

[9] T. Chan, G. Golub and P. Mulet, A Nonlinear Primal-Dual Method for TV-Based Image Restoration, in ICAOS'96, 12th International Conference on Analysis and Optimization of Systems: Images, Wavelets and PDE's, edited by M. Berger, R. Deriche, I. Herlin, J. Jaffre and J.M. Morel, Lecture Notes in Control and Inform. Sci. 219 (1996) 241-252.

[10] J.L. Cox and D.B. Karron, Digital Morse Theory (1998), available at http://www.casi.net

[11] E. De Giorgi and L. Ambrosio, Un nuovo tipo di funzionale del Calcolo delle Variazioni. Atti Accad. Naz. Lincei 8 (1988) 199-210.

[12] A. Desolneux, L. Moisan and J.M. Morel, Edge Detection by Helmholtz Principle. J. Math. Imaging Vision 14 (2001) $271-284$.

[13] F. Dibos and G. Koepfler, Total Variation Minimization by the Fast Level Sets Transform, in Proc. of IEEE Workshop on Variational and Level Sets Methods in Computer Vision (2001).

[14] F. Dibos, G. Koepfler and P. Monasse, Total Variation Minimization: Application to Gray-scale, Color Images and Optical Flow Regularization, in Geometric Level Sets Methods in Imaging, Vision and Graphics, edited by S. Osher and N. Paragios (to appear).

[15] F. Dibos, G. Koepfler and P. Monasse, Image Registration, in Geometric Level Sets Methods in Imaging, Vision and Graphics, edited by S. Osher and N. Paragios (to appear).

[16] S. Durand, F. Malgouyres and B. Rougé, Image Deblurring, Spectrum Interpolation and Application to Satellite Imaging. Math. Model. Numer. Anal. (to appear).

[17] L.C. Evans and R.F. Gariepy, Measure Theory and Fine Properties of Functions. Studies in Advanced Math., CRC Press (1992).

[18] J. Froment, A compact and multiscale image model based on level sets, in Proc. of the $2^{\text {nd }}$ Workshop on Scale-Space Theories in Computer Vision. Corfu, Greece (1999) 152-163.

[19] M. Golubitsky and V. Guillemin, Stable Mappings and Their Singularities. Springer-Verlag (1973).

[20] L. Alvarez, Y. Gousseau and J.M. Morel, Scales in natural images and a consequence on their BV norm, in Proc. of the $2^{\text {nd }}$ Workshop on Scale-Space Theories in Computer Vision. Corfu, Greece (1999) 247-258.

[21] Y. Gousseau and J.M. Morel, Texture Synthesis through Level Sets. Preprint CMLA (2000).

[22] F. Guichard and J.M. Morel, Image iterative smoothing and P.D.E.'s (in preparation).

[23] C. Kuratowski, Topologie I, II. Editions J. Gabay (1992).

[24] J.-L. Lisani, L. Moisan, P. Monasse and J.M. Morel, Affine Invariant Mathematical Morphology Applied to a Generic Shape Recognition Algorithm. Comp. Imaging and Vision 18 (2000).

[25] D. Marr, Vision. Freeman and Co. (1981).

[26] S. Masnou, Filtrage et désocclusion d'images par méthodes d'ensembles de niveau, Ph.D. Thesis. Ceremade, Université ParisDauphine (1998).

[27] F. Meyer and S. Beucher, Morphological Segmentation. J. Visual Commun. Image Representation 1 (1990) 21-46.

[28] J.M. Morel and S. Solimini, Variational methods in image processing. Birkhäuser (1994).

[29] J. Milnor, Morse Theory. Princeton University Press, Annals Math. Studies 51 (1963).

[30] A.S. Kronrod, On functions of two variables. Uspehi Mathematical Sciences (NS) 5 (1950) 24-134. 
[31] S. Mallat, A Wavelet Tour of Signal Processing. Academic Press, New York (1998).

[32] G. Matheron, Random Sets and Integral Geometry. John Wiley, NY (1975).

[33] F. Meyer and P. Maragos, Morphological scale-space representation with levelings, in Proc. of the $2^{\text {nd }}$ Workshop on Scale-Space Theories in Computer Vision (1999) 187-198.

[34] P. Monasse, Contrast invariant image registration, in Proc. of International Conference on Acoustics. Speech and Signal Process. 6 (1999) 3221-3224.

[35] P. Monasse and F. Guichard, Fast computation of a contrast-invariant image representation. IEEE Trans. Image Process. 9 (2000) 860-872.

[36] P. Monasse and F. Guichard, Scale-space from a level lines tree. J. Visual Commun. Image Representation 11 (2000) $224-236$.

[37] D. Mumford and J. Shah, Optimal approximations by piecewise smooth functions and variational problems. Comm. Pure Appl. Math. 42 (1988) 577-685.

[38] M.H.A. Newman, Elements of the Topology of Plane Sets of Points. Dover Publications, New York (1992).

[39] L.I. Rudin, S. Osher and E. Fatemi, Nonlinear Total Variation Based Noise Removal Algorithms. Physica D 60 (1990) $259-269$.

[40] P. Salembier, Morphological multiscale segmentation for image coding. IEEE Trans. Signal Process. 38 (1994) 359-386.

[41] P. Salembier, Region-based filtering of images and video sequences: A morphological viewpoint. Preprint (2000).

[42] P. Salembier and J. Serra, Flat zones filtering, connected operators and filters by reconstruction. IEEE Trans. Image Process. 4 (1995) 1153-1160.

[43] P. Salembier, P. Brigger, J.R. Casas and M. Pardàs, Morphological Operators for Image and Video Compression. IEEE Trans. Image Process. 5 (1996) 881-897.

[44] P. Salembier and L. Garrido, Binary partition tree as an efficient representation for image processing, segmentation, and information retrieval. IEEE Trans. Image Process. 9 (2000).

[45] J. Serra, Image analysis and mathematical morphology. Academic Press (1982).

[46] J. Serra, Image analysis and mathematical morphology. Volume 2: Theoretical Advances. Academic Press (1988).

[47] J. Serra and P. Salembier, Connected operators and pyramids, in Proc. SPIE Image Algebra Math. Morphology. San Diego, CA, SPIE 2030 (1993) 65-76.

[48] G. Sapiro and A. Tannenbaum, On affine plane curve evolution. J. Funct. Anal. 119 (1994) 79-120.

[49] G. Sapiro and A. Tannenbaum, Affine invariant scale space. Int. J. Comput. Vision 11 (1993) 24-44.

[50] C. Vachier, Valuation of image extrema using alterning filters by reconstruction, in Proc. SPIE, Image Algebra and Morphological Processing (1995).

[51] L. Vincent, Grayscale area openings and closings, their efficient implementation and applications, in Proc. of the $1^{\text {st }}$ Workshop on Mathematical Morphology and its Applications to Signal Processing, edited by J. Serra and Ph. Salembrier. Barcelona, Spain (1993) 22-27.

[52] L. Vincent, Morphological area openings and closings for grey-scale images, in Proc. of the Workshop Shape in Picture: Mathematical Description of Shape in Gray-Level Images. Driebergen, The Netherlands (1994) 197-208.

[53] L. Vincent and P. Soille, Watersheds in digital spaces: An efficient algorithm based on immersion simulations. IEEE Trans. Pattern Anal. Machine Intell. 13 (1991) 583-598.

[54] C.R. Vogel and M.E. Oman, Iterative Methods for Total Variation Denoising. SIAM J. Sci. Comput. (to appear).

[55] M. Wertheimer, Untersuchungen zur Lehre der Gestalt, II. Psychologische Forschung 4 (1923) 301-350.

[56] L.P. Yaroslavsky and M. Eden, Fundamentals of digital optics. Birkhäuser, Boston (1996). 